\title{
Pullback attractors of 2D Navier-Stokes equations with weak damping and continuous delay
}

\author{
Juntao Li, Yadi Wang and Xin-Guang Yang*
}

${ }^{*}$ Correspondence:

yangxinguang@hotmail.com College of Mathematics and Information Science, Henan Normal University, Xinxiang, 453007, P.R. China

\begin{abstract}
In this present paper, the existence of pullback attractors for the 2D Navier-Stokes equation with weak damping and continuous delay is considered; by virtue of the classical Galerkin method, we derive the existence and uniqueness of global weak and strong solutions. Using the Aubin-Lions lemma and some energy estimate in the Banach space with delay, we obtain the uniform bound and the existence of a uniform pullback absorbing ball for the solution's semi-processes, and we conclude to the global attractors via verifying the pullback asymptotical compactness by the generalized Arzelà-Ascoli theorem.
\end{abstract}

Keywords: Navier-Stokes equation with weak damping; continuous delay; pullback attractors

\section{Introduction}

In this present paper, we investigate the existence of a pullback attractor for the 2D NavierStokes equations with weak damping and continuous delay that governs the motion of an incompressible fluid:

$$
\left\{\begin{array}{l}
u_{t}-v \Delta u+(u \cdot \nabla) u+\alpha u+\nabla p \\
\quad=f(t-\rho(t), u(t-\rho(t))), \quad(x, t) \in \Omega_{\tau}, \\
\operatorname{div} u=0, \quad(x, t) \in \Omega_{\tau}, \\
u=0, \quad(x, t) \in \partial \Omega_{\tau}, \\
u(\tau, x)=u_{0}(x), \quad x \in \Omega, \\
u(t, x)=\phi(t-\tau, x), \quad(x, t) \in \Omega_{\tau h},
\end{array}\right.
$$

where $\Omega \subset \mathbb{R}^{2}$ is a bounded domain with smooth boundary $\partial \Omega, \Omega_{\tau}=\Omega \times(\tau,+\infty), \Omega_{\tau h}=$ $\Omega \times(\tau-h, \tau), \tau \in \mathbb{R}$ is the initial time, $v$ is the kinematic viscosity of the fluid, $u=u(t, x)=$ $\left(u_{1}(t, x), u_{2}(t, x)\right)$ is the velocity vector field, which is unknown, $p$ is the pressure, $\alpha>0$ is positive constant, $\alpha u$ is the weak damping, $f(t-\rho(t), u(t-\rho(t)))$ is the external force term which contains memory effects during a fixed interval of time of length $h>0, \rho(t)$ is an adequate given delay function, $u_{0}$ is the initial velocity field at the initial time $\tau \in R, \phi$ is the initial state of delay in $[\tau-h, \tau], h>0$ is a constant.

(c) $2015 \mathrm{Li}$ et al. This article is distributed under the terms of the Creative Commons Attribution 4.0 International License (http://creativecommons.org/licenses/by/4.0/), which permits unrestricted use, distribution, and reproduction in any medium, provided you give appropriate credit to the original author(s) and the source, provide a link to the Creative Commons license, and indicate if changes were made. 
When $\alpha=0$ in (1.1), the external force equals 0 , then the system reduces to the wellknown 2D incompressible Navier-Stokes equation:

$$
\begin{aligned}
& v_{t}-v \Delta v+(v \cdot \nabla) \psi+(\psi \cdot \nabla) v+\nabla p=0, \\
& \nabla \cdot v=0 .
\end{aligned}
$$

Since the last century, the global well-posedness and large-time behavior of solutions to the Navier-Stokes equations have attracted many mathematicians.

For more results as regards the well-posedness and long-time behavior of the $2 \mathrm{D}$ autonomous incompressible Navier-Stokes equations, such as the existence of global solutions, the existence global attractors, the Hausdorff dimension, and the inertial manifold approximation, we can refer to Ladyzhenskaya [1], Robinson [2], Sell and You [3], Temam $[4,5]$. Moreover, Caraballo and Real [6-8] derived the existence of a global attractor for the 2D autonomous incompressible Navier-Stokes equation with delays; Chepyzhov and Vishik $[9,10]$ investigated the long-time behavior and convergence of the corresponding uniform (global) attractors for the 2D Navier-Stokes equation with singularly oscillating forces as the external force tending to a steady state by virtue of a linearization method and estimated the corresponding difference equations. Foias and Temam [11,12] gave a survey of the geometric properties of solutions and the connection between solutions, dynamical systems, and turbulence for the Navier-Stokes equations, such as the existence of $\omega$-limit sets; Rosa [13] and Hou and Li [14] obtained the existence of global (uniform) attractors for the $2 \mathrm{D}$ autonomous (non-autonomous) incompressible Navier-Stokes equations in some unbounded domain, respectively; Lu et al. [15] and Lu [16] proved the existence of uniform attractors for 2D non-autonomous incompressible Navier-Stokes equations with normal or less regular normal external force by establishing a new dynamical systems framework; Miranville and Wang [17] derived the attractors for non-autonomous nonhomogeneous Navier-Stokes equations.

For the well-posedness of 3D incompressible Navier-Stokes equations, in 1934, Leray $[18,19]$ derived the existence of a weak solution by a weak convergence method; Hopf [20] improved Leray's result and obtained the familiar Leray-Hopf weak solution in 1951. Since the Navier-Stokes equations lack an appropriate prior estimate and the strong nonlinear property, the existence of a strong solution remains open. For infinite-dimensional dynamical systems, Sell [21] constructed the semiflow generated by the weak solution which lacks the global regularity and obtained the existence of global attractor of the incompressible Navier-Stokes equations on any bounded smooth domain. Cheskidov and Foias [22] introduced a weak global attractor with respect to the weak topology of the natural phase space for a 3D Navier-Stokes equation with periodic boundary; Flandoli and Schmalfuß [23] deduced the existence of weak solutions and attractors for 3D Navier-Stokes equations with a nonregular force; Kloeden and Valero [24] investigated the weak connection of the attainability set of weak solutions of 3D Navier-Stokes equations; Cutland [25] obtained the existence of global solutions for the 3D Navier-Stokes equations with small samples and germs. Chepyzhov and Vishik [26-28] investigated the trajectory attractors for a 3D non-autonomous incompressible Navier-Stokes system based on the work of Leray and Hopf. Using the weak convergence topology of the space $H$ (see below for the definition), Kapustyan and Valero [29] proved the existence of a weak attractor in both autonomous and non-autonomous cases, and gave an existence result of strong attractors. Kapustyan 
et al. [30] considered revised 3D incompressible Navier-Stokes equations generated by an optimal control problem, and they proved the existence of pullback attractors by constructing a dynamical multivalued process.

However, the infinite-dimensional systems for 2D and 3D incompressible Navier-Stokes equations have not yet been completely resolved, so many mathematicians pay attention to this challenging problem, such as the existence of an inertial manifold for 2D incompressible Navier-Stokes equations and the global attractors for the 3D incompressible NavierStokes equations. In this regard, some mathematicians pay attention to the Navier-Stokes equation with weak or strong damping to approximate the standard equations, such as [31-35] for the 2D and 3D incompressible Naver-Stokes equations with damping. However, there are fewer results for the large-time behavior for the Navier-Stokes equations with weak damping and distributed delay. In this paper, we shall show the existence of uniform pullback attractors for the problem (1.1).

This paper will be organized as follows: in Section 2, we shall give some preliminaries; in Section 3, the existence and uniqueness of global weak and strong solutions will be derived; we shall prove the existence of a uniform pullback absorbing ball in Section 4; with the pullback attractors we will conclude in the last section.

\section{Some preliminaries}

In this paper, $C$ will stand for a generic positive constant, depending on $\Omega$ and some constants, but independent of the choice of the initial time $\tau$ and $t$. The Hausdorff semidistance in $X$ from one set $B_{1}$ to another set $B_{2}$ is defined as

$$
\operatorname{dist}_{X}\left(B_{1}, B_{2}\right)=\sup _{b_{1} \in B_{1}} \inf _{b_{2} \in B_{2}}\left\|b_{1}-b_{2}\right\|_{X}
$$

We set $E:=\left\{u \mid u \in\left(C_{0}^{\infty}(\Omega)\right)^{2}, \operatorname{div} u=0\right\}, H$ is the closure of the set $E$ in the $\left(L^{2}(\Omega)\right)^{2}$ topology, $W$ is the closure of the set $E$ in the $\left(H^{2}(\Omega)\right)^{2}$ topology, i.e.,

$$
W=\left\{u \in W\left|\|u\|_{W}=\|u\|_{H^{2}}^{2}, u\right|_{\partial \Omega}=0\right\} .
$$

$P$ is the Helmholz-Leray orthogonal projection in $\left(L^{2}(\Omega)\right)^{2}$ onto the space $H, A:=-P \Delta$ is the Stokes operator subject to the nonslip homogeneous Dirichlet boundary condition with the domain $\left(H^{2}(\Omega)\right)^{2} \cap V$, and $A$ is a self-adjoint positively defined operator on $H$. $A^{-1}$ is a compact operator from $H$ to $H$. The sequence $\left\{\omega_{j}\right\}_{j=1}^{\infty}$ is an orthonormal system of eigenfunctions of $A,\left\{\lambda_{j}\right\}_{j=1}^{\infty}\left(0<\lambda_{1} \leq \lambda_{2} \leq \cdots\right)$ are the eigenvalues of the Stokes operator $A$ corresponding to the eigenfunctions $\left\{\omega_{j}\right\}_{j=1}^{\infty}$. Let

$$
V_{s}:=D\left(A^{\frac{s}{2}}\right), \quad\|V\|_{s}:=\left\|A^{\frac{s}{2}} V\right\|, \quad s \in R,
$$

where $V:=V_{1}=\left(H_{0}^{1}(\Omega)\right)^{2} \cap H$ is a Hilbert space, and $\|v\|_{1}=\|v\|_{V}=\|\nabla v\|$. Clearly, $V_{0}=H$, and $V \hookrightarrow H \equiv H^{\prime} \hookrightarrow V^{\prime} ; H^{\prime}$ and $V^{\prime}$ are dual spaces of $H$ and $V$, respectively, where the injection is dense, continuous. $|\cdot|$ and $(\cdot, \cdot)$ denote the norm and inner product of $H$, i.e.,

$$
(u, v)=\sum_{j=1}^{2} \int_{\Omega} u_{j}(x) v_{j}(x) d x, \quad \forall u, v \in\left(L^{2}(\Omega)\right)^{2} ;
$$


and $\|\cdot\|$ and $((\cdot, \cdot))$ denote the norm and inner product in $V$, i.e.,

$$
((u, v))=\sum_{i, j=1}^{2} \int_{\Omega} \frac{\partial u_{j}}{\partial x_{i}} \cdot \frac{\partial v_{j}}{\partial x_{i}} d x, \quad \forall u, v \in\left(H_{0}^{1}(\Omega)\right)^{2}
$$

and

$$
\|\nabla u\|^{2}:=\sum_{i=1, j=1}^{2}\left\|\partial_{i} u_{j}\right\|_{L^{2}(\Omega)}^{2}, \quad \forall u=\left(u_{1}, u_{2}\right) .
$$

The norm $\|\cdot\|_{*}$ denotes the norm in $V^{\prime},\langle\cdot\rangle$ denotes the dual product in $V$ and $V^{\prime}$.

We define the following bilinear form operator:

$$
B(u, v):=P((u \cdot \nabla) v), \quad \forall u, v \in E,
$$

and the trilinear form operator

$$
b(u, v, w)=\sum_{i, j=1}^{2} \int_{\Omega} u_{i} \frac{\partial v_{j}}{\partial x_{i}} \cdot w_{j} d x=(B(u, v), w) .
$$

Clearly, the trilinear operator satisfies

$$
\begin{aligned}
& b(u, v, v)=0, \quad b(u, v, w)=-b(u, w, v), \quad \forall u, v, w \in V, \\
& \|b(u, v, w)\| \leq C\|u\|^{\frac{1}{2}}\|u\|_{V}^{\frac{1}{2}}\|v\|_{V}\|w\|_{V}, \quad \forall u, v, w \in V, \\
& \|b(u, v, u)\| \leq C\|u\|^{\frac{1}{2}}\|u\|_{V}^{\frac{3}{2}}\|v\|_{V}, \quad \forall u, v \in V, \\
& \|b(u, v, w)\| \leq C\|u\|_{V}\|v\|_{V}\|w\|^{\frac{1}{2}}\|w\|_{V}^{\frac{1}{2}}, \quad \forall u, v, w \in V, \\
& \|b(u, v, w)\| \leq C \lambda_{V}^{\frac{1}{4}}\|u\|_{V}\|v\|_{V}\|w\|_{V}, \quad \forall u, v, w \in V .
\end{aligned}
$$

Next, we introduce some useful inequalities and lemmas.

Young's inequality is

$$
a b \leq \frac{\varepsilon}{p} a^{p}+\frac{1}{q \varepsilon^{\frac{1}{p-1}}} b^{q}, \quad q=\frac{p}{p-1}, 1<p<\infty, \forall a, b, \varepsilon>0 .
$$

The Poincaré inequality is

$$
\|u\| \leq \lambda_{1}^{-\frac{1}{2}}\|u\|_{V}, \quad \forall u \in V
$$

where $\lambda_{1}$ is the first eigenvalue of $A$ under the homogeneous Dirichlet boundary condition.

Lemma 2.1 Let $X=H, V$ or $V^{\prime}$, such that $\|P u\|_{X} \leq\|u\|_{X}$, and $P u \rightarrow u$ in $X$.

Proof See e.g. [6] or [5]. 
Definition 2.1 Let $X$ and $Y$ be Banach spaces, $X \subset Y$, we say that $X$ is compactly embedded in $Y$, written

$$
X \subset \subset Y,
$$

provided

(i) $\|X\|_{Y} \leq C\|X\|_{X}(x \in X)$ for some constant $C$;

(ii) each bounded sequence in $X$ is precompact in $Y$.

Lemma 2.2 (The Lions-Aubin lemma) Let $X \subset \subset H \subset Y$ be Banach spaces; $X$ is the return of itself, if $u_{n}$ is a uniformly bounded sequence in $L^{2}(0, T ; Y)$, and there exists $p>1$, making $\frac{d v_{n}}{d t}$ uniformly bounded in $L^{p}(0, T ; Y)$, such that $u_{n}$ has a subsequence which has strong convergence in $L^{2}(0, T ; H)$.

Proof See e.g. [2] or [5].

Lemma 2.3 (The Gronwall inequality) Let $g, h, y$ all be locally integrable functions in $\left(t_{0},+\infty\right)$ and satisfy

$$
\frac{d y}{d t} \leq g y+h, \quad \forall t \geq t_{0}
$$

$\frac{d y}{d t}$ is locally integrable, and we have

$$
y(t) \leq y\left(t_{0}\right) \exp \left\{\int_{t_{0}}^{t} g(\tau) d \tau\right\}+\int_{t_{0}}^{t} h(s) \exp \left\{-\int_{t}^{s} g(\tau) d \tau\right\} d s, \quad \forall t \geq t_{0} .
$$

Proof See e.g. [36].

Lemma 2.4 (The uniform Gronwall inequality) Let $g(t), h(t)$, and $y(t)$ be three positive locally integrable functions on $\left(t_{0},+\infty\right)$ such that $y(t)$ is locally integrable on $\left(t_{0},+\infty\right)$ and the following inequalities are satisfied:

$$
\begin{aligned}
& \frac{d y}{d t} \leq g y+h, \quad \forall t \geq t_{0}, \\
& \int_{t}^{t+r} g(s) d s \leq a_{1}, \quad \int_{t}^{t+r} h(s) d s \leq a_{2}, \quad \int_{t}^{t+r} y(s) d s \leq a_{3}, \quad \forall t \geq t_{0},
\end{aligned}
$$

where $r, a_{i}(i=1,2,3)$ are positive constants. Then we have

$$
y(t+r) \leq\left(\frac{a_{3}}{r}+a_{2}\right) e^{a_{1}}, \quad \forall t \geq t_{0} .
$$

Proof See e.g. [36].

Lemma 2.5 (The generalized Arzelà-Ascoli theorem) Let $\left\{f_{\gamma}(\theta): \gamma \in \Gamma\right\} \subset C=C([-r, 0]$; $X)$; it is equicontinuous, and for $\forall \theta \in[-r, 0],\left\{f_{\gamma}(\theta): \gamma \in \Gamma\right\}$ has relative compactness in $C([-r, 0] ; X)$. 
Proof See e.g. [8].

Next, we shall give some definitions and a theorem as regards the existence of pullback attractors for non-autonomous systems.

Definition 2.2 Let $X$ be a metric space, the set class $\{U(t, \tau)\}(-\infty<\tau \leq t<+\infty): X \rightarrow X$ is called a process in $X$, if

(i) $U(\tau, \tau) x=x, \tau \in R, \forall x \in X$;

(ii) $U(t, \tau)=U(t, s) U(s, \tau), \forall \tau \leq s \leq t, \tau \in R$.

Let $\mathcal{P}(X)$ denote all the family of nonempty subsets of $X$, and $\mathcal{D}$ the class of all families $\hat{D}=\{D(t) \mid t \in \Omega\} \subset \mathcal{P}(X)$.

Definition 2.3 The process class $\{U(\cdot, \cdot)\}$ is said to be pullback $\mathcal{D}$-asymptotically compact, if for any $t \in R, \hat{D} \in \mathcal{D}$, and $\tau_{n} \rightarrow-\infty, x_{n} \in D\left(\tau_{n}\right)$, the sequence $\left\{U\left(t, \tau_{n}\right) x_{n}\right\}$ possesses a convergence subsequence.

Definition 2.4 A family $B=\{B(t) \mid t \in R\} \in \mathcal{D}$ is said to be pullback $\mathcal{D}$-absorbing if, for each $t \in R$ and $\hat{D} \in \mathcal{D}$, there exists $\tau_{0}(t, \hat{D}) \leq t$ such that

$$
U(t, \tau) D(\tau) \subset B(t), \quad \forall \tau \leq \tau_{0}(t, \hat{D})
$$

Definition 2.5 A family $\hat{A}=\{A(t) \mid t \in R\} \in \mathcal{P}(X)$ is said to be a global pullback $\mathcal{D}$-attractor with respect to the process $\{U(\cdot, \cdot)\}$, if

(i) $A(t)$ is compact for any $t \in R$;

(ii) $\hat{A}$ is pullback $\mathcal{D}$-attracting, i.e.,

$$
\forall \hat{D} \in \mathcal{D}, t \in R, \quad \lim _{\tau \rightarrow-\infty} \operatorname{dist}(U(t, \tau) D(\tau), A(t))=0,
$$

where $\operatorname{dist}\left(C_{1}, C_{2}\right)$ denotes the Hausdorff semi-distance between $C_{1}$ and $C_{2}$ defined as $\operatorname{dist}\left(C_{1}, C_{2}\right)=\sup _{x \in C_{1}} \inf _{y \in C_{2}} d(x, y)$ for $C_{1}, C_{2} \subset X$;

(iii) $\hat{A}$ is invariant, i.e., for all $-\infty<\tau \leq t<+\infty$, we have $U(t, \tau) A(\tau)=A(t)$.

Definition 2.6 We claim that $A(t)=\overline{\bigcup_{\hat{D} \in \mathcal{D}} \Lambda(\hat{D}, t)}, t \in R$, where $\Lambda(\hat{D}, t)$ is defined as

$$
\Lambda(\hat{D}, t)=\bigcap_{s \leq t}\left(\overline{\bigcup_{\tau \leq s} U(t, \tau) D(\tau)}\right), \quad \forall \hat{D} \in \mathcal{D} .
$$

Next we give a result for the existence of a global pullback $\mathcal{D}$-attractor.

Theorem 2.1 (See [31]) Suppose the process $\{U(t, \tau)\}$ is continuous and pullback $\mathcal{D}$-asymptotically compact, and there exists $\hat{B} \in \mathcal{D}$ which is pullback $\mathcal{D}$-absorbing with respect to $\{U(t, \tau)\}$. Then the family $\hat{A}=\{A(t) \mid t \in R\} \subset \mathcal{P}(X), A(t)=\Lambda(\hat{B}, t), t \in R$, is a global pullback $\mathcal{D}$-attractor which is minimal in the sense that if $\hat{C}=\{C(t) \mid t \in R\} \subset \mathcal{P}(X)$ is closed and $\lim _{\tau \rightarrow-\infty} \operatorname{dist}(U(t, \tau) B(\tau), C(t))=0$, then $A(t) \subset C(t)$. 


\section{Existence of global weak and strong solutions}

For each $t \in(\tau, T)$ when $T>\tau$, we define $u:(\tau-h, T) \rightarrow\left(L^{2}(\Omega)\right)^{2}$, here $u_{t}$ is a function in $(-h, 0)$ satisfying $u_{t}=u(t+s), s \in(-h, 0)$.

In the following sections, we denote by $C_{H}=C^{0}([-h, 0] ; H)$ and $C_{V}=C^{0}([-h, 0] ; V)$ two Banach spaces equipped with the norms

$$
\|u\|_{C_{H}}=\sup _{\theta \in[-h, 0]}|u(t+\theta)|
$$

and

$$
\|u\|_{C_{V}}=\sup _{\theta \in[-h, 0]}\|u(t+\theta)\|
$$

respectively, $L_{H}^{2}=L^{2}(-h, 0 ; H), L_{V}^{2}=L^{2}(-h, 0 ; V)$.

Assume that $v_{0} \in H, \eta \in L_{H}^{2}$, then the problems (1.1) can be written in the equivalent form

$$
\begin{aligned}
& \frac{d u}{d t}+v A u+\alpha u+B(u)+\nabla p=f(t-\rho(t), u(t-\rho(t))), \\
& u(\tau)=u_{0}, \quad u(t)=\phi(t), \quad t \in(\tau-h, \tau) .
\end{aligned}
$$

In (1.1), the functions $f:[-h, \infty) \times H \rightarrow H$ and $\phi:[-h, 0] \rightarrow H$ are continuous and satisfy:

(a) $\rho:[0, \infty) \rightarrow[0, h],\left|\frac{d \rho}{d t}\right| \leq M<1$;

(b) $f(t, u)$ satisfies the Lipschitz condition with respect to $u$;

(c) there exist constants $a>0, b>0$ such that $|f(t, u)|^{2} \leq a|u|^{2}+b$;

(d) $\left(v \lambda_{1}\right)^{2}>\frac{a e}{1-M}+\frac{1}{h}, \frac{a}{(1-M) \alpha}>2 v \lambda_{1}$, where $\lambda_{1}$ is the first eigenvalue of $A$ under the homogeneous Dirichlet boundary condition;

(e) from the assumption (d) (i.e., $\left.\left(\nu \lambda_{1}\right)^{2}>\frac{a e}{1-M}+\frac{1}{h}>\frac{a}{1-M}\right)$, we have $-v \lambda_{1}+\frac{a e}{(1-M) \nu \lambda_{1}}<0$, so there exists $\theta>0$, such that $\theta-v \lambda_{1}+\frac{a e}{(1-M) \nu \lambda_{1}}<0$. Noting $\alpha>0$, we can deduce

$$
\theta-v \lambda_{1}-2 \alpha+\frac{a e}{(1-M) \nu \lambda_{1}}<0
$$

(f) from (b), there exists a positive number $L(\beta)$ such that

$$
|f(t, u)-f(t, v)| \leq L(\beta)|u-v|
$$

We shall give the main results in this section.

Theorem 3.1 Let $u_{0} \in H, \phi \in L_{H}^{2}$, the assumptions (a) (f) hold, then there exists a unique global weak solution of (1.1), that satisfies

$$
u \in L^{\infty}(0, T ; H) \cap L^{2}(0, T ; V) .
$$

Proof Assume the orthogonal base in $H$ of $A$ is $w_{j}$ such that $A w_{j}=\lambda_{j} w_{j}$ holds for $j=1,2, \ldots$, $W_{m}=\operatorname{span}\left\{w_{1}, w_{2}, \ldots, w_{m}\right\}$ is the subspace of $H$. Constructing the approximation solution 
$u_{m}(t)=\sum_{j=0}^{n} u_{m k}(t) w_{k}(k=1,2, \ldots, m)$ of problem (1.1), where $u_{m k}(t)$ is to be determined, $u_{m}(t)$ satisfies the approximation equation

$$
\begin{aligned}
& \frac{d u_{m}}{d t}+v A u_{m}+\alpha u_{m}+P_{m} B\left(u_{m}, u_{m}\right)=P_{m} f(t-\rho(t), u(t-\rho(t))), \\
& u_{m}(s)=P_{m} \phi(s), \quad s \in[-h, 0],
\end{aligned}
$$

where $P_{m}: H \rightarrow H$ is the Leray-Helmholtz projection; the pressure $p$ has disappeared by virtue of the application of the $P$.

Next, we shall use the Faedo-Galerkin method to find the global weak solution. We denote $f_{m}=f(t, u(t)), f_{m \rho}=f(t-\rho(t), u(t-\rho(t)))$.

By the local existence of a solution for the ordinary differential equation, we see that the approximation equation of (3.5)-(3.6) possesses a local solution.

Taking the inner product of (3.5) with $u_{m}$ at both sides, using Young's inequality, we obtain

$$
\begin{aligned}
& \frac{1}{2} \frac{d\left|u_{m}\right|^{2}}{d t}+v \lambda_{1}\left|u_{m}\right|^{2}+\alpha\left|u_{m}\right|^{2} \\
& \quad \leq\left|u_{m}\right| \cdot\left|f_{m \rho}\right| \\
& \quad \leq \frac{\alpha\left|u_{m}\right|^{2}}{2}+\frac{\left|f_{m}\right|^{2}}{2 \alpha} \\
& \quad \leq \alpha\left|u_{m}\right|^{2}+\frac{\left|f_{m}\right|^{2}}{2 \alpha} \\
& \quad \leq \alpha\left|u_{m}\right|^{2}+\frac{1}{2 \alpha}\left(a\left|u_{m}(t-\rho(t))\right|^{2}+b\right),
\end{aligned}
$$

i.e.,

$$
\frac{d\left|u_{m}\right|^{2}}{d t} \leq \frac{1}{\alpha}\left(a\left|u_{m}(t-\rho(t))\right|^{2}+b\right)-2 v \lambda_{1}\left|u_{m}\right|^{2}
$$

Integrating $(3.8)$ over $[0, t]$, we derive

$$
\begin{aligned}
\left|u_{m}(t)\right|^{2} & \leq\left|u_{m}(0)\right|^{2}+\frac{b T}{\alpha}+\frac{a}{\alpha} \int_{0}^{t}\left|u_{m}(s-\rho(s))\right|^{2} d s-2 v \lambda_{1} \int_{0}^{t}\left|u_{m}(s)\right|^{2} d s \\
& \leq\left|u_{m}(0)\right|^{2}+\frac{b T}{\alpha}+\frac{a}{\alpha(1-M)} \int_{-h}^{t}\left|u_{m}(r)\right|^{2} d r-2 v \lambda_{1} \int_{0}^{t}\left|u_{m}(s)\right|^{2} d s \\
& \leq K_{0}+K_{1} \int_{-h}^{t}\left|u_{m}(r)\right|^{2} d r-2 v \lambda_{1} \int_{0}^{t}\left|u_{m}(s)\right|^{2} d s,
\end{aligned}
$$

where $K_{0}=\left|u_{m}(0)\right|^{2}+\frac{b T}{\alpha}+K_{1} \int_{-h}^{0}\left|u_{m}(r)\right|^{2} d r, K_{1}=\frac{a}{\alpha(1-M)}, K_{2}=K_{1}-2 v \lambda_{1}$. From (d), $K_{2}=$ $\frac{a}{\alpha(1-M)}-2 v \lambda_{1}>0$.

Hence

$$
\left|u_{m}\right|^{2} \leq K_{0}+\left(K_{1}-2 v \lambda_{1}\right) \int_{0}^{t}\left|u_{m}(s)\right|^{2} d s,
$$

i.e.,

$$
\left|u_{m}\right|^{2} \leq K_{0}+K_{2} \int_{0}^{t}\left|u_{m}(s)\right|^{2} d s
$$


and by the Gronwall inequality, we conclude

$$
\left|u_{m}(t)\right|^{2} \leq K_{0} e^{K_{2} T}
$$

From (3.12) we see that $u_{m}$ is uniformly bounded in $L^{\infty}(0, T ; H) \cap L^{2}(0, T ; V)$.

According to the Alaoglu compact theorem, we can find a subsequence (also denoted as $\left.u_{m}(t)\right)$ such that

$$
\begin{aligned}
& u_{m} \rightarrow^{*} u \in L^{\infty}(0, T ; H) ; \\
& u_{m} \rightarrow u \in L^{2}(0, T ; V),
\end{aligned}
$$

i.e., $u \in L^{\infty}(0, T ; H) \cap L^{2}(0, T ; V)$.

Next, we shall prove $\frac{d u_{m}}{d t}$ is uniformly bounded in $L^{2}\left(0, T ; V^{\prime}\right)$.

Since

$$
\frac{d u_{m}}{d t}=-v A u_{m}-\alpha u_{m}-P_{m} B\left(u_{m}, u_{m}\right)+P_{m} f(t-\rho(t), u(t-\rho(t)))
$$

and $u_{m} \in L^{2}(0, T ; V)$, we have $v A u_{m} \in L^{2}\left(0, T ; V^{\prime}\right)$ and

$$
\begin{aligned}
& \left\|\left(P_{m} B\left(u_{m}, u_{m}\right), u_{m}\right)\right\|_{L^{2}\left(0, T ; V^{*}\right)}^{2} \\
& \quad \leq \int_{0}^{T}\left\|B\left(u_{m}, u_{m}\right)\right\|_{*}^{2} d s=\int_{0}^{T}\left\|\left(u_{m} \cdot \nabla\right) u_{m}\right\|_{*}^{2} d s \\
& \quad \leq c_{5} \int_{0}^{T}\left|u_{m}\right|^{2}\left\|u_{m}\right\|^{2} d s \\
& \quad \leq c_{5}\left\|u_{m}\right\|_{L^{\infty}(0, T ; H)}^{2}\left\|u_{m}\right\|_{L^{2}(0, T ; H)}^{2} \\
& \quad \leq c_{6}\left\|u_{m}\right\|_{L^{\infty}(0, T ; H)}^{2}\left\|u_{m}\right\|_{L^{2}(0, T ; V)}^{2},
\end{aligned}
$$

i.e., $P_{m} B\left(u_{m}, u_{m}\right)$ is uniformly bounded in $L^{2}\left(0, T ; V^{\prime}\right)$, and $P_{m} f(t-\rho(t), u(t-\rho(t))) \in$ $L^{2}(0, T ; V)$ implies $\frac{d u_{m}}{d t}$ is uniformly bounded in $L^{2}\left(0, T ; V^{\prime}\right)$.

In the following, we shall prove the uniqueness of the global solution.

Assume $u(t ; 0, \phi), v(t ; 0, \phi)$ are two solutions of (1.1), whose initial data is $(0, \phi)$; setting $w(t)=u(t)-v(t)$, it follows that

$$
\begin{aligned}
& \frac{d w}{d t}-v \Delta w+B(u, u)-B(v, v)+\alpha w \\
& \quad=f(t-\rho(t), u(t-\rho(t)))-f(t-\rho(t), v(t-\rho(t))) .
\end{aligned}
$$

Noting that

$$
B(u, u)-B(v, v)=B(w, u)+B(u, w)
$$

and

$$
|b(w, u, w)| \leq c_{1}|w|\|w\|\|u\|
$$


taking the inner product of (3.17) with $w$ at both sides, by using Young's inequality, we obtain

$$
\begin{aligned}
& \frac{1}{2} \frac{d|w|^{2}}{d t}+v\|w\|^{2}+\alpha|w|^{2} \\
& \quad \leq|b(w, u, w)|+L(\beta)|w||w(t-\rho(t))| \\
& \quad \leq c_{1}|w|\|w\|\|u\|+L(\beta)|w||w(t-\rho(t))| \\
& \quad \leq \frac{v}{2}\|w\|^{2}+\frac{c_{1}^{2}}{2 v}\|u\|^{2}|w|^{2}+\frac{\alpha|w|^{2}}{2}+\frac{L^{2}(\beta)}{2 \alpha}|w(t-\rho(t))|^{2} \\
& \quad \leq v\|w\|^{2}+\frac{c_{1}^{2}}{v}\|u\|^{2}|w|^{2}+\alpha|w|^{2}+\frac{L^{2}(\beta)}{\alpha}|w(t-\rho(t))|^{2} .
\end{aligned}
$$

Integrating (3.20) over $[0, t]$, and noting

$$
\int_{0}^{t}|w(s-\rho(s))|^{2} d s \leq \frac{1}{1-M} \int_{-h}^{t}|w(s)|^{2} d s,
$$

we get

$$
\begin{aligned}
|w(t)|^{2} \leq & |w(0)|^{2}+\int_{0}^{t} \frac{2 c_{1}^{2}}{v}\|u\|^{2}|w(s)|^{2} d s+\frac{2 L^{2}(\beta)}{(1-M) \alpha} \int_{-h}^{t}|w(s)|^{2} d s \\
= & |w(0)|^{2}+\int_{0}^{t}\left(\frac{2 c_{1}^{2}}{v}\|u\|^{2}+\frac{2 L^{2}(\beta)}{(1-M) \alpha}\right)|w(s)|^{2} d s \\
& +\frac{2 L^{2}(\beta)}{(1-M) \alpha} \int_{-h}^{0}|u(r)-v(r)|^{2} d r
\end{aligned}
$$

since

$$
\int_{-h}^{0}|u(r)-v(r)|^{2} d r=\int_{-h}^{0}|\phi-\phi|^{2} d r=0
$$

we derive

$$
|w(t)|^{2} \leq|w(0)|^{2}+\int_{0}^{t}\left(\frac{2 c_{1}^{2}}{v}\|u\|^{2}+\frac{2 L^{2}(\beta)}{(1-M) \alpha}\right)|w(s)|^{2} d s,
$$

and by the Gronwall inequality, we conclude

$$
|w(t)|^{2} \leq|w(0)|^{2} e^{\int_{0}^{t}\left(\frac{2 c_{1}^{2}}{v}\|u\|^{2}+\frac{2 L^{2}(\beta)}{(1-M) \alpha}\right) d s} .
$$

Theorem 3.1 proves that for $u_{0} \in H, \phi \in L_{H}^{2}$, for the problem (1.1) there exists a unique solution $u_{t}\left(\cdot ; \tau,\left(u_{0}, \phi\right)\right)$. Similar to the construction of a semigroup for an autonomous system, we define the semi-process, the non-autonomous system $\left\{U(t, \tau) \phi: C_{H} \rightarrow C_{H}\right\}$, which satisfies

$$
\begin{aligned}
& U(t, \tau) \phi=u_{t}(\cdot ; \tau,(\phi(0), \phi)), \quad \forall \phi \in C_{H}, t \geq \tau, \\
& U(t, \tau) \phi=I_{d} .
\end{aligned}
$$


Theorem 3.2 Let $u_{0} \in V, \phi \in L_{V}^{2}$, the assumptions (a) (f) hold, then there exists a unique global strong solution of (1.1) which satisfies

$$
u \in L^{\infty}(0, T ; V) \cap L^{2}(0, T ; D(A))
$$

Proof By the local existence of a solution for an ordinary differential equation, we see that the approximation equation of (3.5)-(3.6) possesses a local solution easily, here we omit the details.

Let $u_{m}(t)$ be the approximation solution of (1.1), from Theorem 3.1, there exists a $k=$ $k(T)>0$, such that

$$
\left|u_{m}(t)\right|^{2} \leq k, \quad 0 \leq t \leq T
$$

Define a functional as

$$
W\left(t, u_{m}(t)\right)=\left\|u_{m}(t)\right\|^{2}+\frac{2}{v(1-M)} \int_{t-\rho(t)}^{t}|f(s, u(s))|^{2} d s
$$

differentiating the function $W\left(t, u_{m}(t)\right)$ with respect to $t$, we derive

$$
\begin{aligned}
\frac{d W}{d t} \leq & -2 v\left|A u_{m}\right|^{2}-2 \alpha\left(A u_{m}, u_{m}\right)-2 b\left(u_{m}, u_{m}, A u_{m}\right)+2\left(A u_{m}, f_{\rho}\right) \\
& +\frac{2}{v(1-M)}\left(\left|f_{m}\right|^{2}-\left|f_{m \rho}\right|^{2}\right) \\
\leq & -2 v\left|A u_{m}\right|^{2}-2 \alpha\left\|u_{m}\right\|^{2}+2 c_{1}\left|u_{m}\right|^{\frac{1}{2}}\left\|u_{m}\right\|\left|A u_{m}\right|^{\frac{3}{2}}+2\left(A u_{m}, f_{\rho}\right) \\
& +\frac{2}{v(1-M)}\left(\left|f_{m}\right|^{2}-\left|f_{m \rho}\right|^{2}\right) \\
\leq & -2 v\left|A u_{m}\right|^{2}-2 \alpha \lambda_{1}\left|u_{m}\right|^{2}+2 c_{1}\left|u_{m}\right|^{\frac{1}{2}}\left\|u_{m}\right\|\left|A u_{m}\right|^{\frac{3}{2}}+2\left(A u_{m}, f_{\rho}\right) \\
& +\frac{2}{v(1-M)}\left(\left|f_{m}\right|^{2}-\left|f_{m \rho}\right|^{2}\right)
\end{aligned}
$$

i.e.,

$$
\begin{aligned}
\frac{d W}{d t} \leq & -2 v\left|A u_{m}\right|^{2}+2 \alpha \lambda_{1} k^{2}+\frac{v}{2}\left|A u_{m}\right|^{2}+\frac{128}{\nu^{3}} c_{1}^{4}\left|u_{m}\right|^{2}\left\|u_{m}\right\|^{4}+\frac{\left|f_{\rho}\right|^{2}}{v}+v\left|A u_{m}\right|^{2} \\
& +\frac{2}{v(1-M)}\left(\left|f_{m}\right|^{2}-\left|f_{m \rho}\right|^{2}\right) \\
\leq & -\frac{1}{2} v\left|A u_{m}\right|^{2}+2 \alpha \lambda_{1} k^{2}+\frac{128}{v^{3}} c_{1}^{4}\left|u_{m}\right|^{2}\left\|u_{m}\right\|^{4}+\frac{2}{v(1-M)}\left|f_{m}\right|^{2} \\
& -\frac{1+M}{v(1-M)}\left|f_{m \rho}\right|^{2} \\
\leq & -\frac{1}{2} v\left|A u_{m}\right|^{2}+2 \alpha \lambda_{1} k^{2}+\frac{128}{v^{3}} c_{1}^{4} k^{2}\left\|u_{m}\right\|^{4}+\frac{2}{v(1-M)}\left(a k^{2}+b\right),
\end{aligned}
$$

which implies

$$
\frac{d W}{d t}+\frac{1}{2} \nu|A w|^{2} \leq \frac{128}{\nu^{3}} c_{1}^{4} k^{2}\left\|u_{m}\right\|^{4}+\left(\frac{2 a}{v(1-M)}+2 \alpha \lambda_{1}\right) k^{2}+\frac{2 b}{v(1-M)}
$$


Integrating (3.31) from 0 to $t$ with respect to the time variable, we get

$$
\begin{gathered}
\left\|u_{m}\right\|^{2}+\frac{2}{v(1-M)} \int_{t-\rho(t)}^{t}|f(s, u(s))|^{2} d s+\frac{v}{2} \int_{0}^{t}\left|A u_{m}\right|^{2} d s-W\left(0, u_{m}(0)\right) \\
\leq \frac{128}{v^{3}} c_{1}^{4} k^{2} \int_{0}^{t}\left\|u_{m}\right\|^{4} d s+\left(\frac{2 a}{v(1-M)}+2 \alpha \lambda_{1}\right) T k^{2}+\frac{2 b T}{v(1-M)} .
\end{gathered}
$$

According to the uniform Gronwall inequality, there exists a $R=R(T)$, such that

$$
\left\|u_{m}(t)\right\|^{2} \leq R
$$

From Theorem 3.1, there exists $Q=Q(T)$, such that

$$
\begin{aligned}
& \int_{0}^{T} W\left(s, u_{m}(s)\right) d s \\
& \quad \leq \int_{0}^{T}\left\|u_{m}(s)\right\|^{2} d s+\frac{2}{v(1-M)} \int_{0}^{T} \int_{s-r}^{s}|f(v, u(v))|^{2} d v d s \\
& \quad \leq \int_{0}^{T}\left\|u_{m}(s)\right\|^{2} d s+\frac{2 r T}{v(1-M)}\left(a k^{2}+b\right) \\
& \quad \leq Q .
\end{aligned}
$$

Hence, $u_{m}$ is uniformly bounded in $L^{\infty}(0, T ; V) \cap L^{2}(0, T ; D(A))$, by the structure of the equation, $\frac{d u_{m}}{d t}$ is uniformly bounded in $L^{2}(0, T ; H)$, the proof is similar to Theorem 3.1, here we omit the details. Then there exists $u \in L^{\infty}(0, T ; V) \cap L^{2}(0, T ; D(A))$, such that

$$
\begin{aligned}
& u_{m} \rightarrow^{*} u \quad \text { in } L^{\infty}(0, T ; V) ; \\
& u_{m} \rightarrow u \quad \text { in } L^{2}(0, T ; D(A)) ; \\
& \frac{d u_{m}}{d t} \rightarrow \frac{d u}{d t} \quad \text { in } L^{2}(0, T ; H) .
\end{aligned}
$$

According to the compact embedding theorem, we derive

$$
u_{m} \rightarrow u \quad \text { in } L^{2}(0, T ; V)
$$

The uniqueness of the global solution is similar to Theorem 3.1.

Theorem 3.3 Assume that the assumptions (a) (f) hold, $u_{0} \in H, \phi \in L_{H}^{2}$, the semiprocesses $\left\{U_{f}(t, \tau) \mid t \geq \tau\right\}$ defined by (3.26) is continuous for arbitrary $t \geq \tau$.

Proof Assume $u(t), v(t)$ be two solutions of (1.1), whose initial data is $(\phi(0), \phi),(\psi(0), \psi)$ respectively, setting $w(t)=u(t)-v(t)$, corresponding to the initial data $w(0)=u(0)-v(0)$, it follows that

$$
\begin{aligned}
& \frac{d w}{d t}-v \Delta w+B(u, u)-B(v, v)+\alpha w \\
& \quad=f(t-\rho(t), u(t-\rho(t)))-f(t-\rho(t), v(t-\rho(t))),
\end{aligned}
$$


noting that

$$
B(u, u)-B(v, v)=B(w, u)+B(u, w), \quad|b(w, u, w)| \leq c_{1}|w|\|w\|\|u\|,
$$

taking the inner product of (3.39) with $u_{m}$ at both sides, using Young's inequality, we derive

$$
\begin{aligned}
& \frac{1}{2} \frac{d|w|^{2}}{d t}+v\|w\|^{2}+\alpha|w|^{2} \\
& \quad \leq|b(w, u, w)|+L(\beta)|w||w(t-\rho(t))| \\
& \quad \leq c_{1}|w|\|w\|\|u\|+L(\beta)|w||w(t-\rho(t))| \\
& \quad \leq\left(\frac{v\|w\|^{2}}{2}+\frac{c_{1}^{2}}{2 v}\|u\|^{2}|w|^{2}\right)+\frac{\alpha|w|^{2}}{2}+\frac{L^{2}(\beta)}{2 \alpha}|w(t-\rho(t))|^{2} \\
& \quad \leq v\|w\|^{2}+\frac{c_{1}^{2}}{v}\|u\|^{2}|w|^{2}+\alpha|w|^{2}+\frac{L^{2}(\beta)}{\alpha}|w(t-\rho(t))|^{2},
\end{aligned}
$$

i.e.,

$$
\frac{d|w|^{2}}{d t} \leq \frac{2 c_{1}^{2}}{v}\|u\|^{2}|w|^{2}+\frac{2 L^{2}(\beta)}{\alpha}|w(t-\rho(t))|^{2} .
$$

Integrating (3.42) from 0 to $t$ with respect to the time variable, and noting that

$$
\begin{array}{rl}
\int_{0}^{t}|w(s-\rho(s))|^{2} & d s \leq \frac{1}{1-M} \int_{-h}^{t}|w(s)|^{2} d s \\
|w(t)|^{2}-|w(0)|^{2} & \leq \int_{0}^{t} \frac{2 c_{1}^{2}}{v}\|u\|^{2}|w|^{2} d s+\frac{2 L^{2}(\beta)}{\alpha(1-M)} \int_{-h}^{t}|w(s)|^{2} d s \\
= & \int_{0}^{t} \frac{2 c_{1}^{2}}{v}\|u\|^{2}|w|^{2} d s+\frac{2 L^{2}(\beta)}{\alpha(1-M)} \int_{0}^{t}|w(s)|^{2} d s \\
& +\frac{2 L^{2}(\beta)}{\alpha(1-M)} \int_{-h}^{0}|w(r)|^{2} d r
\end{array}
$$

since

$$
u(t)-v(t)=\phi(t-\tau)-\psi(t-\tau), \quad \tau-h \leq t \leq \tau,
$$

using the formula

$$
\begin{aligned}
\int_{-h}^{0}|w(r)|^{2} d r & =\int_{-h}^{0}|u(r)-v(r)|^{2} d r \\
& \leq\|\phi-\psi\|_{L_{H}^{2}}^{2},
\end{aligned}
$$

we derive

$$
\begin{aligned}
|w(t)|^{2} \leq & |w(0)|^{2}+\frac{2 L^{2}(\beta)}{\alpha(1-M)}\|\phi-\psi\|_{L_{H}^{2}}^{2} \\
& +\int_{0}^{t}\left(\frac{2 c_{1}^{2}}{v}\|u\|^{2}+\frac{2 L^{2}(\beta)}{\alpha(1-M)}\right)|w(s)|^{2} d s,
\end{aligned}
$$


hence, by the Gronwall inequality, we get

$$
\begin{array}{ll}
|w(t)|^{2} \leq\left(|w(0)|^{2}+\frac{2 L^{2}(\beta)}{\alpha(1-M)}\|\phi-\psi\|_{L_{H}^{2}}^{2}\right) e^{\int_{0}^{t}\left(\frac{2 c_{1}^{2}}{v}\|u\|^{2}+\frac{2 L^{2}(\beta)}{\alpha(1-M)}\right) d s}, \quad \forall t \geq \tau-h, \\
\left\|w_{t}\right\|_{C_{H}}^{2} \leq\left(|w(0)|^{2}+\frac{2 L^{2}(\beta)}{\alpha(1-M)}\|\phi-\psi\|_{L_{H}^{2}}^{2}\right) e^{\int_{0}^{t}\left(\frac{2 c_{1}^{2}}{v}\|u\|^{2}+\frac{2 L^{2}(\beta)}{\alpha(1-M)}\right) d s}, \quad \forall t \geq \tau .
\end{array}
$$

The continuous dependence can be obtained obviously.

\section{Existence of pullback absorbing set}

In this section, we shall prove the existence of a pullback absorbing set for the 2D NavierStokes equation with continuous delay and weak damping.

The uniqueness of the solution in Theorem 3.2 proves that the operator $U(t, \tau) \phi$ is a semi-process.

However, we choose the skew-product flow in the space $H \times L_{H}^{2}=M_{H}^{2}$, and define a family of mappings $\tilde{U}(\cdot, \cdot): M_{H}^{2} \rightarrow L_{H}^{2}$, as follows:

$$
\tilde{U}(t, \tau)\left(u_{0}, \phi\right)=u_{t}\left(\cdot ; \tau,\left(u_{0}, \phi\right)\right), \quad \forall\left(u_{0}, \phi\right) \in M_{H}^{2}, t \geq \tau,
$$

obviously,

$$
\tilde{U}(t, \tau) \phi=\tilde{U}(t, \tau)(\phi(0), \phi), \quad t \geq \tau, \phi \in C_{H} .
$$

For arbitrary $\left(u_{0}, \phi\right) \in M_{H}^{2}$, the corresponding norm can be described as

$$
\left\|\left(u_{0}, \eta\right)\right\|_{M_{H}^{2}}^{2}=\left|u_{0}\right|^{2}+\int_{-h}^{0}|\phi(s)|^{2} d s
$$

Lemma 4.1 Assume that $\{B(t)\}_{t \in R}$ are a bounded sets in $C_{H}$, then the mapping $\tilde{U}(\cdot, \cdot)$ is attracting in $C_{H}$, such that $\{B(t)\}_{t \in R}$ for the semi-process $\{U(\cdot, \cdot)\}$ is also attracting in $C_{H}$.

Theorem 4.1 Assume that the assumptions (a) (f) hold, $u_{0} \in H, \phi \in L_{H}^{2}$, the semiprocesses $\{U(t, \tau)\}$ possesses a bounded pullback absorbing set $B_{0}$ in $C_{H}$.

Proof Denote by $D$ a bounded set in $M_{H}^{2}$, then there exists a $d>0$, such that

$$
\left|u_{0}\right|^{2}+\int_{-h}^{0}|\phi(s)|^{2} d s \leq d^{2}
$$

Denote

$$
J\left(t, u_{t}\right)=e^{\theta t}|u(t)|^{2}+\frac{1}{(1-M) v \lambda_{1}} \int_{t-\rho(t)}^{t} e^{\theta s} e^{\theta h}\left|f\left(s, u_{s}\right)\right|^{2} d s
$$

where $\theta$ is an appropriate positive number, satisfying

$$
\theta-v \lambda_{1}-2 \alpha+\frac{a e^{\theta h}}{(1-M) \nu \lambda_{1}}<0 .
$$


Denote $f=f(t, u(t)), f_{\rho}=f(t-\rho(t), u(t-\rho(t)))$, differentiate the function $J\left(t, u_{t}\right)$ with respect to $t$, and we derive

$$
\begin{aligned}
& \frac{d}{d t} J\left(t, u_{t}\right) \leq \theta e^{\theta t}|u|^{2}+2 e^{\theta t}\left(u, \frac{d u}{d t}\right) \\
& +\frac{1}{(1-M) \nu \lambda_{1}}\left[e^{\theta t} e^{\theta h}|f|^{2}-e^{\theta(t-\rho(t))} e^{\theta t}\left|f_{\rho}\right|^{2}\right] \\
& \leq \theta e^{\theta t}|u|^{2}+2 e^{\theta t}\left(u, v \Delta u-\alpha u-B(u, u)+f_{\rho}\right) \\
& +\frac{1}{(1-M) \nu \lambda_{1}}\left[e^{\theta t} e^{\theta h}|f|^{2}-e^{\theta t}\left|f_{\rho}\right|^{2}\right] \\
& \leq \theta e^{\theta t}|u|^{2}-2 \nu \lambda_{1} e^{\theta t}|u|^{2}-2 \alpha e^{\theta t}|u|^{2}+2 e^{\theta t}|u|\left|f_{\rho}\right| \\
& +\frac{1}{(1-M) \nu \lambda_{1}}\left[e^{\theta t} e^{\theta h}|f|^{2}-e^{\theta t}\left|f_{\rho}\right|^{2}\right] \\
& \leq \theta e^{\theta t}|u|^{2}-2 \nu \lambda_{1} e^{\theta t}|u|^{2}-2 \alpha e^{\theta t}|u|^{2}+2 e^{\theta t}\left(\frac{\nu \lambda_{1}|u|^{2}}{2}+\frac{\left|f_{\rho}\right|^{2}}{2 \nu \lambda_{1}}\right) \\
& +\frac{e^{\theta t} e^{\theta h}}{(1-M) \nu \lambda_{1}}|f|^{2}-\frac{e^{\theta t}}{(1-M) \nu \lambda_{1}}\left|f_{\rho}\right|^{2} \\
& \leq \theta e^{\theta t}|u|^{2}-v \lambda_{1} e^{\theta t}|u|^{2}-2 \alpha e^{\theta t}|u|^{2}+\frac{e^{\theta t} e^{\theta h}}{(1-M) \nu \lambda_{1}}|f|^{2} \\
& -\frac{M e^{\theta t}}{(1-M) \nu \lambda_{1}}\left|f_{\rho}\right|^{2} \\
& \leq \theta e^{\theta t}|u|^{2}-v \lambda_{1} e^{\theta t}|u|^{2}-2 \alpha e^{\theta t}|u|^{2}+\frac{e^{\theta t} e^{\theta h}}{(1-M) v \lambda_{1}}\left(a|u|^{2}+b\right) \\
& \leq\left(\theta-v \lambda_{1}-2 \alpha+\frac{a e^{\theta t} e^{\theta h}}{(1-M) \nu \lambda_{1}}\right)|u|^{2} e^{\theta t}+\frac{b e^{\theta t} e^{\theta h}}{(1-M) \nu \lambda_{1}},
\end{aligned}
$$

i.e.,

$$
\frac{d}{d t} J\left(t, u_{t}\right) \leq\left(\theta-\nu \lambda_{1}-2 \alpha+\frac{a e^{\theta t} e^{\theta h}}{(1-M) \nu \lambda_{1}}\right)|u|^{2} e^{\theta t}+\frac{b e^{\theta t} e^{\theta h}}{(1-M) \nu \lambda_{1}}
$$

Since

$$
b(u, u, u)=0, \quad 2|u|\left|f_{\rho}\right| \leq v \lambda_{1}|u|^{2}+\frac{\left|f_{\rho}\right|^{2}}{v \lambda_{1}}
$$

integrating (4.8) from $\tau$ to $t$ with respect to time variable, combining (a) $\sim(e)$, we obtain

$$
\begin{aligned}
e^{\theta t}|u(t)|^{2} \leq & \frac{1}{(1-M) \nu \lambda_{1}} \int_{\tau-\rho(t)}^{\tau} e^{\theta s} e^{\theta h}\left|f\left(s, u_{s}\right)\right|^{2} d s \\
& +\left(\theta-\nu \lambda_{1}-2 \alpha+\frac{a e^{\theta h}}{(1-M) \nu \lambda_{1}}\right) \int_{\tau}^{t}|u(s)|^{2} e^{\theta s} d s \\
& +\frac{b e^{\theta h}}{(1-M) \nu \lambda_{1}} \int_{\tau}^{t} e^{\theta s} d s+e^{\theta \tau}\left|u_{0}\right|^{2} \\
\leq & \frac{1}{(1-M) \nu \lambda_{1}} \int_{-h}^{0} e^{\theta s} e^{\theta h}\left|f\left(s, u_{s}\right)\right|^{2} d s
\end{aligned}
$$




$$
\begin{aligned}
& +\left(\theta-\nu \lambda_{1}-2 \alpha+\frac{a e^{\theta h}}{(1-M) \nu \lambda_{1}}\right) \int_{\tau}^{t}|u(s)|^{2} e^{\theta s} d s \\
& +\frac{b e^{\theta h}}{(1-M) \nu \lambda_{1}} \frac{e^{\theta t}-e^{\theta \tau}}{\theta}+e^{\theta \tau}\left|u_{0}\right|^{2}
\end{aligned}
$$

here $\theta-v \lambda_{1}-2 \alpha+\frac{a e^{\theta h}}{(1-M) \nu \lambda_{1}}<0$, hence

$$
\begin{aligned}
e^{\theta t}|u(t)|^{2} \leq & e^{\theta \tau}\left|u_{0}\right|^{2}+\frac{1}{(1-M) \nu \lambda_{1}} \int_{-h}^{0} e^{\theta s} e^{\theta h}\left|f\left(s, u_{s}\right)\right|^{2} d s \\
& +\frac{b e^{\theta h}}{(1-M) \theta \nu \lambda_{1}}\left(e^{\theta t}-e^{\theta \tau}\right), \quad t \geq \tau,
\end{aligned}
$$

choosing $\sigma \in[-h, 0]$, substituting for $t: t+\sigma$, we have

$$
\begin{aligned}
e^{\theta(t-h)}|u(t+\sigma)|^{2} \leq & e^{\theta \tau}\left|u_{0}\right|^{2}+\frac{1}{(1-M) \nu \lambda_{1}} \int_{-h}^{0} e^{\theta s} e^{\theta h}\left|f\left(s, u_{s}\right)\right|^{2} d s \\
& +\frac{b e^{\theta h}}{(1-M) \theta v \lambda_{1}}\left(e^{\theta(t+\sigma)}-e^{\theta \tau}\right)
\end{aligned}
$$

i.e.,

$$
\begin{aligned}
e^{\theta t}|u(t+\sigma)|^{2} \leq & e^{\theta h}\left(e^{\theta \tau}\left|u_{0}\right|^{2}+\frac{1}{(1-M) \nu \lambda_{1}} \int_{-h}^{0} e^{\theta s} e^{\theta h}\left|f\left(s, u_{s}\right)\right|^{2} d s\right) \\
& +\frac{b e^{\theta h}}{(1-M) \theta \nu \lambda_{1}} e^{\theta h}\left(e^{\theta t}-e^{\theta \tau}\right)
\end{aligned}
$$

hence

$$
\begin{aligned}
e^{\theta t}|u(t+\sigma)|^{2} \leq & e^{\theta h}\left(e^{\theta \tau}\left|u_{0}\right|^{2}+\frac{1}{(1-M) \nu \lambda_{1}} \int_{-h}^{0} e^{\theta s} e^{\theta h}\left|f\left(s, u_{s}\right)\right|^{2} d s\right) \\
& +\frac{b e^{2 \theta h}}{(1-M) \theta \nu \lambda_{1}} e^{\theta t} \\
\leq & C_{1}+C_{2} e^{\theta t}
\end{aligned}
$$

where

$$
\begin{aligned}
& C_{1}=e^{\theta h}\left(e^{\theta \tau}\left|u_{0}\right|^{2}+\frac{1}{(1-M) \nu \lambda_{1}} \int_{-h}^{0} e^{\theta s} e^{\theta h}\left|f\left(s, u_{s}\right)\right|^{2} d s\right), \\
& C_{2}=\frac{b e^{2 \theta h}}{(1-M) \theta v \lambda_{1}} .
\end{aligned}
$$

By the Gronwall inequality, we get

$$
e^{\theta t}\left|u_{t}\right|^{2} \leq C_{1}+C_{2} e^{\theta t}
$$

Combining (4.14)-(4.16), we conclude

$$
\left\|u_{t}\right\|_{C_{H}}^{2} \leq C_{1} e^{-\theta t}+C_{2} \quad(t \geq \tau+h)
$$


substituting for $\tau: t-s$, denoting $u(\cdot, \cdot)$ as $u\left(\cdot ; t-s,\left(u_{0}, \phi\right)\right)$ also for arbitrary $t, s \geq h$, we have

$$
\begin{aligned}
\left\|u_{t}\right\|_{C_{H}}^{2} & =\left\|\tilde{U}(t, t-s)\left(u_{0}, \phi\right)\right\|_{C_{H}}^{2} \\
& \leq e^{\theta h}\left(e^{\theta(t-s)}\left|u_{0}\right|^{2}+\frac{1}{(1-M) \nu \lambda_{1}} \int_{-h}^{0} e^{\theta s} e^{\theta h}\left|f\left(s, u_{s}\right)\right|^{2} d s\right) e^{-\theta t}+C_{2} \\
& \leq e^{\theta h}\left(e^{-\theta s}\left|u_{0}\right|^{2}+\frac{e^{-\theta t} e^{\theta h}}{(1-M) v \lambda_{1}} \int_{-h}^{0} e^{\theta s}\left|f\left(s, u_{s}\right)\right|^{2} d s\right)+C_{2} \\
& \leq e^{\theta h}\left(e^{-\theta s} d^{2}+\frac{e^{\theta h}}{(1-M) v \lambda_{1}} \int_{-h}^{0} e^{\theta s}\left|f\left(s, u_{s}\right)\right|^{2} d s\right)+C_{2} .
\end{aligned}
$$

Denoting

$$
\tilde{\rho}^{2}=\frac{e^{2 \theta h}}{(1-M) \nu \lambda_{1}} \int_{-h}^{0} e^{\theta s}\left|f\left(s, u_{s}\right)\right|^{2} d s+C_{2}, \quad 2 \tilde{\rho}^{2}=\tilde{\rho}_{H}^{2},
$$

then for some $\tilde{T}_{D}(t) \geq h$, such that $\left\|\tilde{U}(t, t-s)\left(u_{0}, \phi\right)\right\|_{C_{H}} \leq \tilde{\rho}_{H}$ for $s \geq \tilde{T}_{D}(t)$, there exists a ball $B_{C_{H}}\left(0, \tilde{\rho}_{H}\right)$ for the semi-process $\tilde{U}(t, t-s)\left(u_{0}, \phi\right), B$ is a pullback absorbing set.

From Lemma 4.1, the ball $B_{C_{H}}\left(0, \tilde{\rho}_{H}\right)$ for the semi-process $\{U(t, t-s) \phi\}$ is also a pullback absorbing set, which completes the proof.

Theorem 4.2 Assume that the assumptions in Theorem 4.1 hold, there exists a bounded pullback attracting set for the semi-process $\{U(\cdot, \cdot)\}$ in $C_{V}$.

Proof Let

$$
Q\left(t, u_{t}\right)=|u|^{2}+\frac{1}{(1-M) \alpha} \int_{t-\rho(t)}^{t}|f(s, u(s))|^{2} d s .
$$

Differentiate the function $Q\left(t, u_{t}\right)$ with respect to $t$, and we derive

$$
\begin{aligned}
\frac{d Q}{d t}= & 2\left(u, \frac{d u}{d t}\right)+\frac{1}{(1-M) \alpha}\left(|f|^{2}-\left|f_{\rho}\right|^{2}\right) \\
\leq & 2\left(u, v u-\alpha u-B(u, u)+f_{\rho}\right)+\frac{1}{(1-M) \alpha}\left(|f|^{2}-\left|f_{\rho}\right|^{2}\right) \\
\leq & -2 v\|u\|^{2}+2|u|\left|f_{\rho}\right|-2 \alpha|u|^{2}+\frac{1}{(1-M) \alpha}\left(|f|^{2}-\left|f_{\rho}\right|^{2}\right) \\
\leq & -2 v\|u\|^{2}+2 \alpha|u|^{2}+\frac{\left|f_{\rho}\right|^{2}}{2 \alpha}-2 \alpha|u|^{2}+\frac{1}{(1-M) \alpha}|f|^{2} \\
& -\frac{1}{(1-M) \alpha}\left|f_{\rho}\right|^{2} \\
\leq & -2 v\|u\|^{2}+\frac{1}{(1-M) \alpha}|f|^{2}-\frac{1+M}{2(1-M) \alpha}\left|f_{\rho}\right|^{2} \\
\leq & -2 v\|u\|^{2}+\frac{1}{(1-M) \alpha}|f|^{2} \\
\leq & -2 v\|u\|^{2}+\frac{1}{(1-M) \alpha}\left(a|u|^{2}+b\right) .
\end{aligned}
$$


From (4.11) and (4.17), there exist $T>0, \delta_{1}>0$, we have $\max \left\{|u|^{2},\left|u_{t}\right|^{2}\right\} \leq \delta_{1}^{2}$, for $t>T$. Integrating (4.21) from $t$ to $t+r$ with respect to the time variable, we obtain

$$
\begin{aligned}
& Q(t+r)+2 v \int_{t}^{t+r}\|u\|^{2} d s \\
& \quad \leq Q\left(t, u_{t}\right)+\frac{a}{(1-M) \alpha} \delta_{1}^{2} r+\frac{b r}{(1-M) \alpha} \\
& \quad \leq|u|^{2}+\frac{1}{(1-M) \alpha} \int_{t-\rho(t)}^{t}\left(a|u|^{2}+b\right) d s+\frac{a \delta_{1}^{2} r}{(1-M) \alpha}+\frac{b r}{(1-M) \alpha},
\end{aligned}
$$

hence

$$
2 v \int_{t}^{t+r}\|u\|^{2} d s \leq\left(1+\frac{(r+h) a}{(1-M) \alpha}\right) \delta_{1}^{2}+\frac{(r+h) b}{(1-M) \alpha} .
$$

From (4.23), we have

$$
\int_{t}^{t+r}\|u\|^{2} d s \leq \delta_{2}^{2}
$$

here

$$
\delta_{2}^{2}=\frac{1}{2 v}\left[\left(1+\frac{(r+h) a}{(1-M) \alpha}\right) \delta_{1}^{2}+\frac{(r+h) b}{(1-M) \alpha}\right] .
$$

\section{Denoting}

$$
W\left(t, u_{t}\right)=\|u\|^{2}+\frac{2}{(1-M) v} \int_{t-\rho(t)}^{t}|f(s, u(s))|^{2} d s
$$

we have

$$
\begin{aligned}
\int_{t}^{t+r} W d s & =\int_{t}^{t+r}\|u\|^{2} d s+\frac{2}{(1-M) v} \int_{t}^{t+r}\left(\int_{s-\rho(s)}^{s}\left(a|u|^{2}+b\right) d r\right) d s \\
& \leq r \delta_{2}^{2}+\frac{2 r h}{(1-M) v}\left(a \delta_{1}^{2}+b\right) \\
& =\delta_{3} .
\end{aligned}
$$

Differentiate the function $W$ with respect to $t$, and combining with the Young's inequality, we get

$$
\begin{aligned}
\frac{d W}{d t}= & \frac{d\|u\|^{2}}{d t}+\frac{2}{(1-M) v}\left(|f|^{2}-\left|f_{\rho}\right|^{2}\right) \\
\leq & -2 v|A u|^{2}-2|(A u, \alpha u)|+2|b(u, u, A u)|+2\left(A u, f_{\rho}\right) \\
& +\frac{2}{(1-M) v}\left(|f|^{2}-\left|f_{\rho}\right|^{2}\right) \\
\leq & -2 v|A u|^{2}-2 \alpha\|u\|^{2}+2 c_{1}|u|^{\frac{1}{2}}\|u\||A u|^{\frac{3}{2}}+2|A u|\left|f_{\rho}\right| \\
& +\frac{2}{(1-M) v}\left(|f|^{2}-\left|f_{\rho}\right|^{2}\right)
\end{aligned}
$$




$$
\begin{aligned}
\leq & -2 v|A u|^{2}-2 \alpha\|u\|^{2}+2\left(\frac{v}{3}|A u|^{2}+\frac{27}{v^{3}} c_{1}^{4}|u|^{2}\|u\|^{4}\right)+v|A u|^{2}+\frac{\left|f_{\rho}\right|^{2}}{v} \\
& +\frac{2}{(1-M) v}\left(|f|^{2}-\left|f_{\rho}\right|^{2}\right) \\
\leq & -\frac{v}{3}|A u|^{2}-2 \alpha\|u\|^{2}-\frac{1+M}{(1-M) v}\left|f_{\rho}\right|^{2}+\frac{54}{v^{3}} c_{1}^{4}|u|^{2}\|u\|^{4}+\frac{2|f|^{2}}{(1-M) v} \\
\leq & \frac{54}{v^{3}} c_{1}^{4}|u|^{2}\|u\|^{4}+\frac{2|f|^{2}}{(1-M) v} \\
\leq & \frac{54}{v^{3}} c_{1}^{4} \delta_{1}^{2}\|u\|^{4}+\frac{2}{(1-M) v}\left(a|u|^{2}+b\right) .
\end{aligned}
$$

If we denote

$$
\begin{aligned}
& a_{1}=\frac{54}{v^{3}} c_{1}^{4} \delta_{1}^{2} \delta_{2}^{2} r \\
& a_{2}=\frac{2}{(1-M) v}\left(a|u|^{2}+b\right) r \\
& a_{3}=\delta_{3}
\end{aligned}
$$

by the uniform Gronwall inequality, it follows that

$$
W \leq\left(\frac{a_{3}}{r}+a_{2}\right) e^{a_{1}} \quad(t \geq h+r)
$$

Noting that $\|u\|^{2} \leq W\left(t, u_{t}\right)$, using a similar technique to Theorem 4.1 , we easily get

$$
\|u\|_{C_{V}}^{2} \leq\left(\frac{a_{3}}{r}+a_{2}\right) e^{a_{1}} \quad(t \geq h+r)
$$

substituting for $\tau: t-s$, denoting $u(\cdot, \cdot)$ as $u\left(\cdot ; t-s,\left(u_{0}, \phi\right)\right)$, for arbitrary $t, s \geq h$, we derive

$$
\left\|\tilde{U}(t, t-s)\left(u_{0}, \phi\right)\right\|_{C_{V}}^{2} \leq\left(\frac{a_{3}}{r}+a_{2}\right) e^{a_{1}} \quad\left(t \geq \tilde{T}_{D}(t)+h+r\right)
$$

here $\tilde{\rho}_{V}^{2}=\left(\frac{a_{3}}{r}+a_{2}\right) e^{a_{1}}, B_{C_{V}}\left(0, \tilde{\rho}_{V}\right)$ is a bounded pullback attracting set for the semiprocesses $\{U(\cdot, \cdot)\}$ in $C_{V}$.

\section{Existence of pullback attractors in $\boldsymbol{H}$}

The main results in our paper can be stated as follows.

Theorem 5.1 Assume that (a) (f) hold, $u_{0} \in H, \phi \in L_{H}^{2}$, there exists a pullback attractor $\mathcal{A}$ of the problem (1.1) for the solutions' semi-process $\left\{U_{f}(t, \tau) \mid t \geq \tau\right\}$.

Proof Theorem 4.1 guarantees that there exists a bounded attracting set of the problem (1.1), and Theorem 4.2 proves that the problem (1.1) possesses a bounded attracting set in $C_{V}$, respectively. If we can prove $u_{t}$ is compact in $C_{H}$, then the problem (1.1) possesses a pullback attractor; this is equivalent to proving the next two properties by the generalized Arzelà-Ascoli theorem: 
(1) $V \subset \subset H$ is compact.

(2) $\{U(t, \tau)\}$ is equicontinuous.

We have

$$
\left|u\left(t ; t+\theta_{1}, \phi\right)-u\left(t ; t+\theta_{2}, \phi\right)\right|=\left|\int_{t+\theta_{1}}^{t+\theta_{2}} u^{\prime}(r) d r\right|
$$

and we get

$$
\begin{aligned}
& \left|u\left(t ; t+\theta_{1}, \phi\right)-u\left(t ; t+\theta_{2}, \phi\right)\right| \\
& \quad \leq \int_{t+\theta_{1}}^{t+\theta_{2}}\left|u^{\prime}(r)\right| d r \\
& \quad \leq \int_{t+\theta_{1}}^{t+\theta_{2}}\left(\left|f_{\rho}\right|+v|A u|+\alpha|u|+|B(u)|\right) d r \\
& \quad \leq \int_{t+\theta_{1}}^{t+\theta_{2}}\left(\frac{\left|f_{\rho}\right|^{2}}{2 v}+\frac{v}{2}+\frac{v}{2}+\frac{v^{2}|A u|^{2}}{2 v}+\frac{v}{2}+\frac{\alpha^{2}|u|^{2}}{2 v}+c_{1}|A u|\|u\|\right) d r \\
& \quad \leq \int_{t+\theta_{1}}^{t+\theta_{2}}\left(\frac{\left|f_{\rho}\right|^{2}}{2 v}+\frac{v}{2}+\frac{v}{2}+\frac{v|A u|^{2}}{2}+\frac{v}{2}+\frac{\alpha^{2}\|u\|^{2}}{2 v \lambda_{1}}+\frac{c_{1}^{2}\|u\|^{2}}{2 v}+\frac{v|A u|^{2}}{2}\right) d r \\
& \quad \leq \int_{t+\theta_{1}}^{t+\theta_{2}}\left(\frac{\left|f_{\rho}\right|^{2}}{v}+v+v+v|A u|^{2}+v+\frac{\alpha^{2}\|u\|^{2}}{v \lambda_{1}}+\frac{c_{1}^{2}\|u\|^{2}}{v}+v|A u|^{2}\right) d r \\
& \quad=\int_{t+\theta_{1}}^{t+\theta_{2}}\left(\frac{\left|f_{\rho}\right|^{2}}{v}+3 v+2 v|A u|^{2}+\frac{\alpha^{2}\|u\|^{2}}{v \lambda_{1}}+\frac{c_{1}^{2}\|u\|^{2}}{v}\right) d r .
\end{aligned}
$$

Taking the inner product of (3.3) with $A u$ at both sides, we obtain

$$
\begin{aligned}
& \frac{1}{2} \frac{d\|u\|^{2}}{d t}+v|A u|^{2}+\alpha\|u\|^{2}+b(u, u, A u) \\
& \quad=\left(f_{\rho}, A u\right) \\
& \quad=\left|f_{\rho}\right| \cdot|A u| \\
& \quad \leq \frac{\left|f_{\rho}\right|^{2}}{2 v}+\frac{v|A u|^{2}}{2},
\end{aligned}
$$

such that

$$
\int_{t+\theta_{1}}^{t+\theta_{2}} v|A u|^{2} d r \leq \frac{1}{v} \int_{t+\theta_{1}}^{t+\theta_{2}}\left|f_{\rho}\right|^{2} d r+\left\|u\left(t+\theta_{1}\right)\right\|^{2}
$$

and substituting (5.4) into (5.2), we get

$$
\begin{aligned}
& \left|u\left(t+\theta_{1}\right)-u\left(t+\theta_{2}\right)\right| \\
& \quad \leq \int_{t+\theta_{1}}^{t+\theta_{2}}\left[\frac{2\left|f_{\rho}\right|^{2}}{v}+3 v+\left(\frac{\alpha^{2}}{v \lambda_{1}}+\frac{c_{1}^{2}}{v}+1\right)\|u\|^{2}\right] d r \\
& \quad \leq\left(3 v+\frac{\alpha^{2}+\lambda_{1} c_{1}^{2}+v \lambda_{1}}{v \lambda_{1}}\|u\|^{2} \tilde{\rho}_{V}^{2}\right)\left|\theta_{1}-\theta_{2}\right|+\frac{2}{v} \int_{t+\theta_{1}}^{t+\theta_{2}}\left|f_{\rho}\right|^{2} d r .
\end{aligned}
$$


Hence, $U$ is equicontinuous, and compactness is proved.

From the fundamental theory of the existence of the pullback attractor generated by the problem (1.1), one completes the proof.

\section{Competing interests}

The authors declare that they have no competing interests.

\section{Authors' contributions}

The authors declare that the work was realized in collaboration with the same responsibility. All authors read and approved the final manuscript.

\section{Acknowledgements}

This paper was in part supported by NSFC (No. 61203293), Foundation of Henan Educational Committee (2011B120005), Key Scientific and Technological Project of Henan Province (122102210131), College Young Teachers Program of Henan Province (2012GGJS-063), the Young Backbone Teachers Fund of Henan Normal University (5101019470605), the Program for Science and Technology Innovation Talents in University of Henan Province (No. 13HASTIT040) and the Foundation of Henan Educational Committee (No. 14B1 10029), Program for Innovative Research Team (in Science and Technology) in University of Henan Province (14IRTSTHN023).

\section{Received: 3 November 2014 Accepted: 4 May 2015 Published online: 21 May 2015}

\section{References}

1. Ladyzhenskaya, OA: The Mathematical Theory of Viscous Incompressible Flow. Gordon \& Breach, New York (1969)

2. Robinson, JC: Infinite-Dimensional Dynamical Systems. Cambridge University Press, Cambridge (2001)

3. Sell, GR, You, Y: Dynamics of Evolutionary Equations. Springer, New York (2002)

4. Temam, R: Navier-Stokes Equations: Theory and Numerical Analysis. North-Holland, Amsterdam (1979)

5. Temam, R: Infinite Dimensional Dynamical Systems in Mechanics and Physics, 2nd edn. Springer, Berlin (1997)

6. Caraballo, T, Real, J: Navier-Stokes equations with delays. R. Soc. Lond. Proc., Ser. A, Math. Phys. Eng. Sci. 457, 2441-2453 (2001)

7. Caraballo, T, Real, J: Asymptotic behavior for two-dimensional Navier-Stokes equations with delays. R. Soc. Lond. Proc., Ser. A, Math. Phys. Eng. Sci. 459, 3181-3194 (2003)

8. Caraballo, T, Real, J: Attractors for 2D Navier-Stokes models with delays. J. Differ. Equ. 205, 271-297 (2004)

9. Chepyzhov, W, Vishik, Ml: Averaging of 2D Navier-Stokes equations with singularly oscillating forces. Nonlinearity 22, 351-370 (2009)

10. Vishik, Ml, Chepyzhov, W: The global attractor of the nonautonomous 2D Navier-Stokes system with singularly oscillating external force. Dokl. Math. 75(2), 236-239 (2007)

11. Foias, C, Temam, R: The connection between the Navier-Stokes equations, dynamical systems and turbulence. In: Directions in Partial Differential Equations, pp. 55-73. Academic Press, New York (1987)

12. Foias, C, Temam, R: Some analytic and geometric properties of the solutions of the Navier-Stokes equations. J. Math Pures Appl. 58(3), 339-368 (1979)

13. Rosa, R: The global attractor for the 2D Navier-Stokes flow on some unbounded domains. Nonlinear Anal. TMA 32(1), 71-85 (1998)

14. Hou, Y, Li, K: The uniform attractors for the 2D non-autonomous Navier-Stokes flow in some unbounded domain. Nonlinear Anal. TMA 58, 609-630 (2004)

15. $L u, S, W u, H, Z$ hong, C: Attractors for nonautonomous 2D Navier-Stokes equations with normal external forces. Discrete Contin. Dyn. Syst. 13(3), 701-719 (2005)

16. Lu, S: Attractors for nonautonomous 2D Navier-Stokes equations with less regular normal forces. J. Differ. Equ. 230, 196-212(2006)

17. Miranville, A, Wang, X: Attractors for nonautonomous nonhomogeneous Navier-Stokes equations. Nonlinearity 10(5), 1047-1061 (1997)

18. Leray, J: Essai sur les mouvements plans d'un liquide visqueux que limitent des parois. J. Math. Pures Appl. 13, 331-418 (1934)

19. Leray, J: Essai sur le mouvement d'un liquide visqueux emplissant l'espace. Acta Math. 63, 193-248 (1934)

20. Hopf, E: Über die Anfangswertaufgable für die hydrodynamischen Grundgleichungen. Math. Nachr. 4, 213-231 (1951)

21. Sell, GR: Global attractors for the three-dimensional Navier-Stokes equations. J. Dyn. Differ. Equ. 8, 1-33 (1996)

22. Cheskidov, A, Foias, C: On global attractors of the 3D Navier-Stokes equations. J. Differ. Equ. 231, 714-754 (2006)

23. Flandoli, F, Schmalfuß, B: Weak solutions and attractors for three-dimensional Navier-Stokes equations with nonregular force. J. Dyn. Differ. Equ. 11(2), 355-398 (1999)

24. Kloeden, PE, Valero, J: The weak connectedness of the attainability set of weak solutions of the three-dimensional Navier-Stokes equations. Proc. R. Soc. A 463, 1491-1508 (2007)

25. Cutland, NJ: Global attractors for small samples and germs of 3D Navier-Stokes equations. Nonlinear Anal. TMA 62, 265-281 (2005)

26. Chepyzhov, W, Vishik, Ml: Evolution equations and their trajectory attractors. J. Math. Pures Appl. 76, 664-913 (1997)

27. Chepyzhov, VV, Vishik, MI: Attractors for Equations of Mathematical Physics. Am. Math. Soc., Providence (2001)

28. Vishik, Ml, Chepyzhov, W: Trajectory and global attractors of three-dimensional Navier-Stokes systems. Math. Notes 71, 177-193 (2002)

29. Kapustyan, OV, Valero, J: Weak and strong attractors for the 3D Navier-Stokes system. J. Differ. Equ. 240, 249-278 (2007)

30. Kapustyan, OV, Kasyanov, PO, Valero, J: Pullback attractors for a class of extremal solutions of the 3D Navier-Stokes system. J. Math. Anal. Appl. 373, 535-547 (2011) 
31. Cai, X, Jiu, Q: Weak and strong solutions for the incompressible Navier-Stokes equations with damping. J. Math. Anal. Appl. 343, 799-809 (2008)

32. Kalantarov, VK, Titi, ES: Global attractors and determining models for the 3D Navier-Stokes-Voight equations. Chin. Ann. Math., Ser. B 30, 697-714 (2009)

33. Qin, Y, Yang, $X, L i u, X$ : Averaging of a 3D Navier-Stokes-Voight equation with singularly oscillating forces. Nonlinear Anal., Real World Appl. 13, 893-904 (2012)

34. Song, X, Hou, Y: Attractors for the three-dimensional incompressible Navier-Stokes equations with damping. Discrete Contin. Dyn. Syst. 31(1), 239-252 (2011)

35. Yang, $X, L i, J$ : Upper semi-continuity of uniform attractors for the $2 D$ non-autonomous Navier-Stokes equations with damping. Abstr. Appl. Anal. 2013, Article ID 861292 (2013)

36. Lakshmikantham, V, Leela, S, Martynyuk, AA: Stability Analysis of Nonlinear Systems. Monographs and Textbooks in Pure and Applied Mathematics, vol. 125. Dekker, New York (1989). ISBN:0-8247-8067-1

Submit your manuscript to a SpringerOpen ${ }^{\circ}$ journal and benefit from:

- Convenient online submission

Rigorous peer review

- Immediate publication on acceptance

- Open access: articles freely available online

- High visibility within the field

- Retaining the copyright to your article 